\title{
Análisis de costo-efectividad de dos intervenciones para el control de la malaria en el área urbana de Buenaventura, Colombia
}

\author{
Sandra Lorena Girón ${ }^{1,2}$, Julio César Mateus ${ }^{1,2}$, Carlos Enrique Castellar ${ }^{3}$ \\ ${ }^{1}$ División Salud, Fundación FES Social, Cali, Colombia. \\ ${ }^{2}$ Escuela de Salud Pública, Universidad del Valle, Cali, Colombia. \\ ${ }^{3}$ Facultad de Ciencias Sociales y Económicas, Universidad del Valle, Cali, Colombia.
}

Introducción. Se compararon los costos y resultados de dos alternativas para el control de la malaria en el área urbana de Buenaventura, en la costa Pacífica colombiana. Una constituida por las actividades del Programa Nacional, la otra por la integración al programa de una estrategia educativa llamada Alternativa Integrada.

Objetivo. Evaluar la costo-efectividad de dos alternativas de intervención para el control de la malaria en el área urbana de Buenaventura.

Materiales y métodos. Se realizó un análisis de costo-efectividad, desde los puntos de vista institucional y familiar. Los costos institucionales se obtuvieron revisando los registros de las instituciones responsables de la implementación de cada alternativa. Los costos familiares se obtuvieron a través de entrevistas. La medida de efectividad fue el número de casos evitados por cada 10.000 habitantes.

Resultados. Los costos institucionales del Programa Nacional y de la Alternativa Integrada por cada 10.000 habitantes fueron $\$ 5.374 .082$ (US\$3,766) y $\$ 34 ' 808.525$ (US\$24,392,8), respectivamente. Los costos familiares promedio en la zona donde operó el Programa Nacional fueron \$51.657 (US\$36.2), mientras que donde operó la Alternativa Integrada fueron \$40.527 (US\$28.4). El número de casos evitados por cada 10.000 habitantes fue $12,9\left(\mathrm{Cl}_{95 \%}-6,0 ; 31,8\right)$ para la zona del Programa Nacional y $264,6\left(\mathrm{Cl}_{95 \%} 254,1 ; 275,1\right)$ para la zona de la Alternativa Integrada. Las razones de costo-efectividad institucional del Programa Nacional y de la Alternativa Integrada fueron $\$ 417.254,8$ (US\$292.4) y $\$ 131.569,4(U S \$ 92.2)$, respectivamente. Conclusión. La integración de las actividades de la estrategia educativa a las del Programa Nacional fue la alternativa más costo-efectiva. Los datos sugieren que la estrategia educativa debe ser integrada a las actividades del Programa Nacional.

Palabras clave: análisis costo-efectividad, evaluación económica, malaria, promoción de la salud, prevención primaria, educación en salud.

Cost-effectiveness analysis of two strategies for malaria control in the urban area of Buenaventura, Colombia

Introduction. Costs and results of two alternatives for malaria control were compared. One constituted by the activities of the National Programme, the other by the integration of an educational strategy denominated "Integrated Alternative" (IA) into the national program in Buenaventura on the Pacific Coast of Colombia.

Objective. To evaluate the cost-effectiveness of two alternatives for malaria control in the urban area of Buenaventura.

Materials and methods. A cost-effectiveness analysis was carried out from an institutional and household perspective. Institutional costs were obtained by reviewing records of institutions that implemented each alternative; household costs were obtained from interviews. Effectiveness measurement was the number of averted cases per 10.000 inhabitants.

Results. Institutional costs of National Programme and Integrated Alternative were US $\$ 3,766$ and US $\$ 24,932.8$ respectively. Average household cost in the zone where National Programme was implemented was US\$36.2, while in the zone where IA was applied it was US\$28.4. The 
number of averted cases per 10,000 inhabitants was $12.9\left(\mathrm{Cl}_{95 \%}-6.0 ; 31.8\right)$ for National Programme and $264.6\left(\mathrm{Cl}_{95 \%} 254.1 ; 275.1\right)$ for Integrated Alternative. The institutional costeffectiveness ratios of National Programme and Integrated Alternative were US\$292.4 and US\$92.2, respectively.

Conclusion. Integration of the educational strategy into the National Programme was the most cost-effective alternative. Our data suggest that the educational strategy should be added to the National Programme activities.

Key words: cost-benefit analysis, malaria, health promotion, primary prevention, health education.

La malaria es uno de los principales problemas de salud pública en la costa del Pacífico colombiano. Esta región, con solo 2,5\% de la población colombiana, agrupa $10 \%$ a $15 \%$ de los casos de malaria, de los cuales $60 \%$ a $85 \%$ se debe a Plasmodium falciparum (1), parásito que produce una enfermedad más grave y de mayor fatalidad.

La incidencia de malaria en 2000 y 2001 fue de 3,9 y 2,2 casos por cien mil habitantes, respectivamente (2,3). Desde 1987, se ha documentado un proceso de urbanización de la malaria en Buenaventura. Más de 44\% de los casos en 1991 ocurrió en áreas urbanas, porcentaje que se redujo a 20\% en 1998 (4) después de la implementación de dos intervenciones orientadas a detener el proceso.

Desde 1947, el Programa Nacional de Enfermedades Tropicales ha realizado actividades de promoción, prevención, diagnóstico y tratamiento para el control de la malaria en toda el área de Buenaventura. Además, en 1994 se estableció una estrategia educativa basada en la participación comunitaria en las áreas de mayor incidencia de la enfermedad. Por tanto, dos alternativas para el control de la malaria han operando simultáneamente en el área urbana de Buenaventura. Una caracterizada por las actividades desarrolladas exclusivamente por el Programa Nacional, y la otra por la integración de la estrategia educativa al programa.

Una evaluación de efectividad de la estrategia educativa encontró que las familias que tuvieron

\footnotetext{
Correspondencia:

Sandra Lorena Girón, Calle 23N No. 5 AN-47

Barrio Versalles, Cali, Valle, Colombia.

Tel. 57-2-6534141 Fax: 57-2-6534141

sandragiron@fundacionfes.org
}

Recibido: 10/04/06; aceptado: 27/07/06 contacto con la estrategia educativa tenían mayores conocimientos acerca del zancudo, la eliminación de criaderos, el uso de toldillos y la fumigación que las no cubiertas por la estrategia (Alvarado B, Alzate A. Evaluación de una estrategia de fortalecimiento en participación comunitaria, para el control de la malaria en área urbana de Buenaventura 1997. Informe final. Centro de Investigaciones en Epidemiología. Universidad del Valle, Cali, 1998. Observaciones no publicadas).

Dado que se ha demostrado la efectividad de la estrategia educativa, y que la implementación de acciones simultáneas para el control de la malaria genera un consumo sustancial de recursos, el objetivo de este estudio fue establecer la costoefectividad de dos alternativas para el control de la malaria, desde el punto de vista institucional y familiar, con el fin de generar información para la toma de decisiones relacionadas con la prevención de la enfermedad y la asignación de recursos para su control.

\section{Materiales y métodos \\ Área}

El estudio se realizó en el área urbana de Buenaventura, principal puerto y centro urbano de la costa del Pacífico colombiano. El puerto está ubicado a 57 metros sobre el nivel del mar, en una región con condiciones climáticas que favorecen la transmisión de malaria durante todo el año, con una temperatura promedio de $28^{\circ} \mathrm{C}$ y pluviosidad promedio anual de $6.980 \mathrm{~mm}$. El área urbana de Buenaventura está compuesta de 12 comunas que agrupan $85 \%$ de la población total del puerto (295.000 habitantes, aproximadamente).

\section{Tipo de estudio}

Se realizó un análisis de costo-efectividad desde el punto de vista institucional y familiar (5), 
comparando los costos y resultados de dos alternativas para el control de la malaria en el área urbana de Buenaventura.

\section{Descripción de las alternativas}

Una alternativa se caracteriza por las actividades del Programa Nacional, consistentes en fumigación, rociamiento, impregnación de toldillos, eliminación de criaderos y entrega de información acerca del control de factores ambientales. El programa también ofrece gratuitamente el diagnóstico de malaria y su tratamiento. En nuestro artículo, dicha alternativa se denomina Programa Nacional.

La otra alternativa se caracteriza por la integración de una estrategia educativa a las actividades del Programa Nacional. La estrategia educativa se basó en la participación comunitaria, y se realizó aplicando una metodología de organización y movilización social para el control de la enfermedad. Con el apoyo de líderes comunitarios, la población recibió educación orientada a mejorar los conocimientos acerca del reconocimiento temprano de los síntomas y de las medidas de prevención y control de la malaria. Denominamos esta opción como Alternativa integrada.

\section{Costos institucionales}

Se identificaron, cuantificaron y valoraron los costos de cada alternativa de acuerdo a las actividades desarrolladas entre 1993 y 1998 por el Programa Nacional y por la Alternativa Integrada (5).

Dado que los recursos invertidos en cada alternativa no sólo fueron monetarios, y algunos de ellos se usaron en más de una actividad, fue necesario aplicar técnicas de valoración (5). Por tanto, se utilizaron los precios de mercado del recurso humano, áreas físicas, vehículos, equipos y suministros para estimar el costo de oportunidad de dichos recursos.

\section{Costos familiares}

Para la medición de los costos familiares directos e indirectos originados antes y después del diagnóstico, se realizaron entrevistas a personas que solicitaban el diagnóstico de malaria. Las entrevistas buscaban medir los costos ocasionados por las actividades previas y posteriores al diagnóstico orientadas a la atención de los síntomas (5). Los costos previos al diagnóstico incluyeron los originados desde el inicio de los síntomas hasta la fecha de diagnóstico (realizado por gota gruesa). Los costos posteriores al diagnóstico incluyeron los producidos desde el diagnóstico hasta el día en que el paciente retornó a las actividades normales. Si el paciente no se recuperaba en los ocho días posteriores al diagnóstico, sólo se tenían en cuenta los costos generados hasta ese día.

Como costos directos se incluyeron los costos de transporte, consulta, diagnóstico, compra de medicamentos y otro tratamiento no provisto por el Programa Nacional. Los costos indirectos incluyeron aquellos originados en la pérdida de ingresos debido a incapacidad laboral del paciente y a los días dedicados por los cuidadores. La pérdida de ingreso se valoró con el salario mínimo legal vigente colombiano como aproximación al costo de oportunidad del tiempo productivo perdido.

\section{Medida de efectividad}

La medida de efectividad de cada alternativa fue el número de casos evitados por cada 10.000 habitantes. A partir de registros epidemiológicos, se obtuvo el número de casos reportados para cada comuna durante el periodo anterior a la implementación de la Alternativa Integrada (1987 a 1994), y se construyó una serie histórica de casos mensuales por cada 10.000 habitantes para cada semana epidemiológica, usando las cifras reportadas por el Censo Nacional para Buenaventura (6). Las series se modelaron mediante regresión lineal simple y se calculó el número de casos esperados para cada zona. Se realizaron estimaciones para el periodo de operación simultánea de las dos alternativas (1995 a 1998) bajo el supuesto de que si la Alternativa Integrada no se hubiera implementado, la relación de casos entre las series se habría mantenido. Se controló la autocorrelación entre las series mediante la aplicación del método de máxima verosimilitud de Cochrane-Orcut (7). Finalmente, se estimó el número de casos evitados por cada 10.000 habitantes con base en la diferencia entre el número de casos esperados y el número de casos registrados en cada zona. 


\section{Recolección de datos}

Los datos de los costos institucionales de cada alternativa se recolectaron utilizando formatos diseñados, probados y ajustados antes de su aplicación. Los datos de costos para el periodo 1993-1998 se obtuvieron a través de la revisión de archivos de las instituciones que implementaron cada alternativa. Para estimar los costos de recursos no monetarios, se realizaron entrevistas al personal que participó en la aplicación de cada alternativa.

Para recolectar los datos de costos familiares originados ante un caso de malaria, se entrevistaron individuos que presentaban síntomas de la enfermedad y que solicitaban el diagnóstico en los principales centros de diagnóstico de la ciudad. Después de obtener el consentimiento informado, se entrevistó a pacientes y acompañantes con el fin de reconstruir los procesos y los costos de la búsqueda de atención. Además, se concertó una segunda entrevista a realizarse en el domicilio del paciente ocho días más tarde para reconstruir los costos generados por el cuidado del paciente después de obtenido el diagnóstico.

\section{Análisis}

Los datos de costos se midieron en pesos colombianos; se eliminó el efecto de la inflación para obtener cifras comparables a través de todo el periodo y se convirtieron a pesos constantes de 1998.

Se calcularon los costos institucionales por cada 10.000 habitantes. Para obtener las razones de costo-efectividad institucional, los costos se dividieron por el número de casos evitados por cada 10.000 habitantes en cada alternativa. Las razones de costo-efectividad se compararon calculando la razón de costo-efectividad incremental.

Partiendo de los intervalos de confianza de los casos evitados en cada alternativa, se realizó un análisis de sensibilidad de las razones de costoefectividad con el fin de evaluar la consistencia de los resultados.

Se calcularon los costos familiares promedio y se compararon con la prueba de Kruskal-Wallis (8).

\section{Resultados}

\section{Número de casos reportados y evitados}

Antes de la implementación simultánea de las alternativas (1987 a 1994), por cada caso en la zona donde operó el Programa Nacional hubo 1,82 (IC 95\% 1,49 a 2,15) casos en la zona donde se aplicó la Alternativa Integrada. Después de la implementación (1995 a 1998), esta razón se redujo a 0,56 (IC 95\% 0,46 a 0,66).

El número de casos evitados por cada 10.000 habitantes en la zona de Programa Nacional fue 12,9 (IC 95\% -6,0 a 31,8), mientras que en la zona de Alternativa Integrada fue de 264,6 (IC 95\% 254,1 a 275,1 ) casos.

\section{Razones de costo-efectividad institucional}

La alternativa del Programa Nacional fue menos costosa que la Alternativa Integrada desde el punto de vista de las instituciones, pero la Alternativa Integrada presentó una menor razón de costo-efectividad, o sea $\$ 131.569,4$ (US $\$ 92,2$ ), por caso evitado por cada 10.000 habitantes.

El costo adicional generado por la integración de las actividades de la estrategia educativa a las del Programa Nacional para evitar un caso adicional por cada 10.000 habitantes fue de $\$ 116.871$ (US \$81,9) (cuadro 1).

\section{Análisis de sensibilidad}

Los cuadros 2 y 3 presentan las razones de costoefectividad y costo-efectividad incremental cuando el número de casos evitados varía de acuerdo a los límites inferiores y superiores de los intervalos de confianza. Para cada escenario, la alternativa más costo-efectiva fue la Alternativa Integrada.

En la situación menos deseable, cuando la Alternativa Integrada (Al) evita 254,1 casos y cabe la posibilidad de que el Programa Nacional no evite ninguno, el costo adicional de evitar otro caso (costo incremental) con la Al fue de $\$ 113.161$ (US \$79.3). En el mejor escenario, cuando las dos alternativas evitan la mayor cantidad de casos, el costo adicional por caso adicional evitado con la A I fue de $\$ 121.010$ (US \$84.8).

\section{Costos familiares}

Se realizaron 1.970 entrevistas, 34\% de los pacientes tuvo diagnóstico positivo para malaria. 
Cuadro 1. Razones de costo-efectividad y costo-efectividad incremental según alternativa.

\begin{tabular}{lccccc}
\hline Alternativa & $\begin{array}{c}\text { Costo institucional } \\
\text { por } \mathbf{1 0 . 0 0 0} \text { hab. }^{*}\end{array}$ & $\begin{array}{c}\text { Casos evitados/ } \\
\mathbf{1 0 . 0 0 0} \text { hab. }\end{array}$ & (B) & $\begin{array}{c}\text { (A/B) } \\
\text { Razón de } \\
\text { costo-efectividad }\end{array}$ \\
\hline Alternativa Integrada & US $\$ 24,392.8$ & (A1) & 264,6 & (B1) & US $\$ 92.2$ \\
Programa Nacional & US $\$ 3,766.2$ & (A2) & \multicolumn{1}{c}{12,9} & (B2) & US $\$ 292.4$ \\
& Costo-efectividad incremental por 10,000 habitantes & (A1-A2) / (B1-B2) & US $\$ 81.9$ \\
\hline
\end{tabular}

US $\$=1.427$ pesos colombianos de 1998 .

Cuadro 2. Razones de costo-efectividad institucional según alternativa y escenarios de casos evitados (CE).

\begin{tabular}{|c|c|c|c|c|c|}
\hline \multicolumn{3}{|c|}{ Alternativa Integrada } & \multicolumn{3}{|c|}{ Programa Nacional } \\
\hline $\begin{array}{l}\text { Bajo } \\
(\mathrm{CE}=254,1)\end{array}$ & $\begin{array}{l}\text { Promedio } \\
(\mathrm{CE}=264,6)\end{array}$ & $\begin{array}{l}\text { Alto } \\
(\mathrm{CE}=275,1)\end{array}$ & $\begin{array}{l}\text { Bajo } \\
(\mathrm{CE}=-6,0)\end{array}$ & $\begin{array}{l}\text { Promedio } \\
(\mathrm{CE}=12,9)\end{array}$ & $\begin{array}{l}\text { Alto } \\
(\mathrm{CE}=31,8)\end{array}$ \\
\hline US $\$ 96.02$ & US $\$ 92.2$ & US $\$ 88.6$ & -US $\$ 624.6$ & US \$292.4 & US $\$ 118.4$ \\
\hline
\end{tabular}

1 US $\$=1.427$ pesos colombianos de 1998 .

Cuadro 3. Razones de costo-efectividad incremental según alternativa y escenarios de casos evitados (CE).

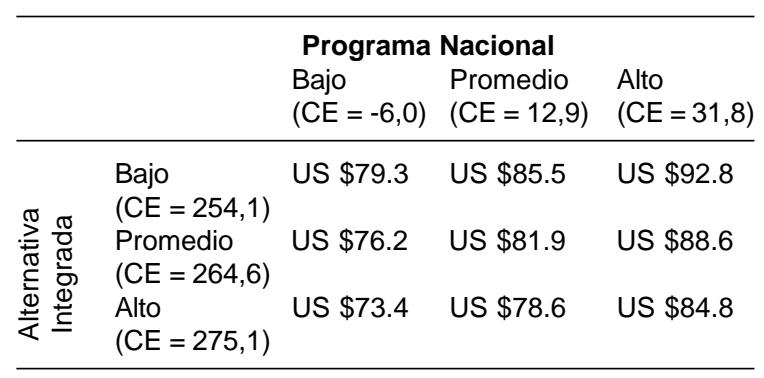

1 US $\$=1.427$ pesos colombianos de 1998 .

Los resultados aquí presentados se concentran en este grupo de pacientes. Las familias de la zona donde se aplicó la Alternativa Integrada tuvieron costos indirectos pre y posdiagnóstico menores, así como costos totales posdiagnóstico y costos totales menores comparadas con las familias de la zona donde se aplicó sólo el Programa Nacional. Las familias de la zona de la Alternativa Integrada asumieron mayores costos comparadas con las familias de la zona del Programa Nacional. Las familias residentes en la zona donde se aplicó la Alternativa Integrada asumieron mayores costos directos prediagnóstico comparadas con las familias de la zona donde operó el Programa Nacional (cuadro 4).

\section{Discusión}

En este estudio se encontró que en el área urbana de Buenaventura, la integración del
Programa Nacional y una estrategia educativa basada en participación comunitaria fue la alternativa más costo-efectiva para el control de la malaria.

Desde el punto de vista institucional, esta conclusión se basa en varios argumentos. Primero, aunque la opción del Programa Nacional fue menos costosa, existe la posibilidad de que el número de casos evitados sea cero. Segundo, la Alternativa Integrada alcanzó el mayor número de casos evitados, aproximadamente 265 por 10.000 habitantes, aunque los costos fueron cinco veces mayores. Tercero, la razón de costo-efectividad de la Alternativa Integrada fue más baja que la del Programa Nacional, y, cuarto, la razón de costoefectividad incremental indica que evitar un caso adicional de malaria con la Alternativa Integrada costaría \$116.871(US \$81.9), el cual es un costo razonable para evitar un caso de malaria.

Estos resultados se mantuvieron aún en el peor escenario, cuando el número de casos evitados para cada una de las alternativas fue el menor. Incluso en esta situación, las razones de costoefectividad de la Alternativa Integrada fueron menores, y el costo adicional de evitar un caso adicional ( $\$ 113.161$, US $\$ 79.3$ ) podría considerarse razonable.

Por tanto, es posible sugerir que cuando las actividades de control de la malaria centradas en el control de factores ambientales tales como 
Cuadro 4. Comparación de costos familiares por caso de malaria según alternativa y tipo de costos (US dólares de 1998).

\begin{tabular}{|c|c|c|c|c|c|c|c|}
\hline \multirow[b]{2}{*}{ Costo } & \multicolumn{3}{|c|}{ Alternativa Integrada } & \multicolumn{3}{|c|}{ Programa Nacional } & \multirow[b]{2}{*}{$p^{*}$} \\
\hline & Mediana & Rango & $\begin{array}{c}\text { Suma de } \\
\text { rangos }\end{array}$ & Mediana & Rango & $\begin{array}{c}\text { Suma de } \\
\text { rangos }\end{array}$ & \\
\hline \multicolumn{8}{|l|}{ Prediagnóstico } \\
\hline $\begin{array}{l}\text { Directo } \\
\text { Indirecto } \\
\text { Total prediagnóstico }\end{array}$ & $\begin{array}{c}2,1 \\
13,4 \\
15,5\end{array}$ & $\begin{array}{l}0-130,3 \\
0-200,4 \\
0-247,1\end{array}$ & $\begin{array}{l}81,9 \\
74,2 \\
74,8\end{array}$ & $\begin{array}{r}2,0 \\
13,4 \\
20,0\end{array}$ & $\begin{array}{l}0-126,8 \\
0-113,6 \\
0-140,2\end{array}$ & $\begin{array}{l}72,4 \\
80,0 \\
79,4\end{array}$ & $\begin{array}{l}0,0155 \\
0,0431 \\
0,0930\end{array}$ \\
\hline \multicolumn{8}{|l|}{ Posdiagnóstico } \\
\hline $\begin{array}{l}\text { Directo } \\
\text { Indirecto } \\
\text { Total posdiagnóstico } \\
\text { Total }\end{array}$ & $\begin{array}{c}0 \\
0 \\
0,3 \\
28,4\end{array}$ & $\begin{array}{c}0-78,9 \\
0-93,5 \\
0-132,3 \\
0-287,2\end{array}$ & $\begin{array}{l}77,3 \\
73,7 \\
72,9 \\
73,1\end{array}$ & $\begin{array}{r}0 \\
6,7 \\
7,6 \\
36,2\end{array}$ & $\begin{array}{l}0-136,8 \\
0-267,2 \\
0-277,7 \\
0-316,7\end{array}$ & $\begin{array}{l}76,9 \\
80,5 \\
81,4 \\
81,1\end{array}$ & $\begin{array}{l}0,8176 \\
0,0207 \\
0,0053 \\
0,0077\end{array}$ \\
\hline
\end{tabular}

* Valores de $p$ basados en la prueba de Kruskal-Wallis. 1 US $\$=1.427$ pesos colombianos de 1998.

rociamiento y eliminación de criaderos, se realizan con educación y participación comunitaria orientada a incrementar los conocimientos sobre la enfermedad puede esperarse una reducción de la incidencia de la enfermedad a costos razonables.

Estudios previos han sugerido que la educación en salud es una estrategia efectiva para el control de la malaria (9-12).

En Buenaventura, una evaluación de la efectividad de la estrategia educativa, en la cual se realizó una comparación con el Programa Nacional, encontró que la educación brindada por la estrategia mejoró los conocimientos, las actitudes y las prácticas frente a la malaria (Alvarado B, Alzate A. Evaluación de una estrategia de fortalecimiento en participación comunitaria para el control de la malaria en el área urbana de Buenaventura 1997. Informe final. Centro de Investigaciones en Epidemiología. Universidad del Valle, Cali, 1998. Observaciones no publicadas).

Desde el punto de vista familiar, las familias ubicadas en la zona intervenida con el Programa Nacional tuvieron costos $27 \%$ mayores para atender un caso de malaria comparadas con las residentes en el área intervenida con la Alternativa Integrada. Esto sugiere que la educación para el control de la malaria contribuye a que las familias gasten menos recursos, quizá porque los síntomas pueden reconocerse de forma más temprana, acuden más rápido a los centros de diagnóstico y, por tanto, se genera menor pérdida de ingresos por los días de incapacidad laboral.
Los menores costos posdiagnóstico asumidos por familias de la zona intervenida con la Alternativa Integrada sugieren que una vez se reconoce la enfermedad, y dado que el tratamiento es gratuito, las familias gastan menos en solucionar el problema de salud.

Los costos familiares en la zona intervenida con la Alternativa Integrada (\$40.527, US \$28.4) y en la intervenida con el Programa Nacional ( $\$ 51.657$, US \$36.2) reportados en este estudio son sustancialmente mayores que los descritos en Sri Lanka (US \$15.6 por año, US \$7) $(13,14)$, Rwanda (US \$11.8) (15), Burkina Faso (US \$5.9) (16) y Ghana (US \$8.7) (17). Esta diferencia puede deberse a hechos relacionados con los estudios mencionados: primero, las investigaciones referenciadas fueron realizadas en áreas rurales donde los niveles de ingreso per cápita restringen los gastos de búsqueda de atención; segundo, la reconstrucción de los costos incluyó periodos de hasta un mes, lo cual puede producir una subestimación de los resultados, y, tercero, en algunos lugares no se contaba con métodos para confirmar un posible caso de malaria, por tanto los costos medidos podrían incluir los generados por otro tipo de enfermedad generadora de menores costos.

De igual manera, los costos aquí reportados son mayores que los reportados en 1994 para la costa Pacífica colombiana y ecuatoriana, donde el costo promedio de un caso de malaria fue de US $\$ 17.3$ y US $\$ 10.4$, respectivamente (18). 
Dado que la Alternativa Integrada tiene un menor costo por caso evitado y que los costos familiares ante un caso de malaria fueron menores en el área intervenida con la Alternativa Integrada, es aconsejable que las instituciones responsables del control de la malaria realicen acciones no sólo para el control de factores ambientales sino también de educación de la población en riesgo. Por tanto, es posible decir que la integración de las actividades del Programa Nacional y las de la estrategia educativa no sólo permite mejorar los niveles de conocimientos, actitudes y prácticas y contribuye a evitar casos, sino que también permite un uso más eficiente de recursos institucionales y familiares.

Los cambios en la incidencia de malaria en el área donde se implementó la Alternativa Integrada pueden atribuirse en gran parte a las acciones conjuntas del Programa Nacional y la estrategia educativa por varias razones. Primero, las zonas intervenidas presentan condiciones climáticas similares dada su proximidad; segundo, las estimaciones se basaron en registros del sistema de vigilancia, el cual realizó los mismos procedimientos durante el periodo estudiado, y, tercero, la población urbana de Buenaventura en su conjunto comparte condiciones socioeconómicas similares y es relativamente homogénea en su desarrollo urbano (6).

Una de las fortalezas de esta investigación es haber realizado el análisis desde el punto de vista institucional y familiar, teniendo en cuenta que es posible que una alternativa pueda ser costoefectiva para las instituciones, pero no para las familias, y viceversa.

Otra es la medición detallada de los costos institucionales, los cuales incluyeron no sólo recursos monetarios, sino también aquellos no monetarios, tales como el tiempo del personal y el uso de espacios. Además, los costos familiares incluyeron costos directos de los pacientes y costos de los cuidadores, y aquellos generados por pérdida de tiempo laboral.

Las debilidades de este estudio residen en la posible subestimación de los costos institucionales, debido principalmente a que algunas de las fuentes de información (archivos institucionales) presen- taban problemas de calidad. Respecto a los costos familiares, también es posible que los datos estén subestimados debido a la recolección retrospectiva de los datos. Sin embargo, si tal subestimación realmente existió, puede asumirse que fue igual para todos los pacientes y que fue pequeña dado el corto periodo de tiempo a recordar (desde el momento de aparición de los síntomas hasta el diagnóstico y ocho días después del diagnóstico).

Además, es importante tener en cuenta que la mayoría de la población de Buenaventura es de bajos a medianos ingresos y, por tanto, tendería a recordar fácilmente y con razonable precisión los costos adicionales generados por una enfermedad.

La relevancia de los resultados reportados en este estudio radica en la posibilidad de influir en quienes toman decisiones en relación con la asignación de recursos para la prevención y control de la malaria. De esta manera, la decisión de integrar la estrategia educativa a las actividades del Programa Nacional generaría una asignación costo-efectiva de recursos, tanto para las instituciones como para las familias. De acuerdo con los resultados de esta evaluación, dicha decisión conllevaría una disminución del número de casos de malaria, aumentaría los conocimientos de la población frente a las medidas de prevención y disminuiría los costos familiares ante la presencia de casos de malaria. Es preciso tener en cuenta que si la estrategia educativa se integra a las actividades del Programa Nacional, éste solo tendría que asumir costos de implementación, debido a que los costos de diseño, validación y ajuste ya habrían sido sufragados por las instituciones que desarrollaron la estrategia.

\section{Agradecimientos}

Los autores agradecen la colaboración de los funcionarios encargados de realizar las actividades de diseño e implementación, y de los pacientes y sus familias por la información suministrada para la realización del proyecto.

\section{Conflicto de intereses}

Sandra Girón y Julio Mateus son investigadores de la Fundación FES Social, una de las instituciones que diseñó e implementó la 
estrategia educativa. Sin embargo, ingresaron a la institución después de la finalización de dichas actividades y, por tanto, no participaron ni en el diseño ni en la implementación de la estrategia educativa. La Secretaría Departamental de Salud del Valle del Cauca es otra de las instituciones que participó en el diseño e implementación de la estrategia educativa, pero el interventor asignado al proyecto de evaluación no participó en las mencionadas actividades.

\section{Financiación}

Este estudio fue financiado con recursos de la Secretaría Departamental de Salud del Valle del Cauca, Colombia. Contrato de Cooperación No.099-00 de 2000.

\section{Referencias}

1. Carrasquilla $\mathbf{G}$, Banguero $\mathbf{M}$, Sánchez $\mathbf{P}$, Carvajal F, Barker RH Jr, Gervais GW, et al. Epidemiologic tools for malaria surveillance in an urban setting of low endemicity along the Colombian Pacific coast. Am J Trop Med Hyg 2000;62:132-7.

2. Méndez F, Carrasquilla G. Epidemiología de la malaria en el área urbana de Buenaventura: análisis de la ocurrencia en el periodo 1987-1993. Colomb Med 1995;26:77-85.

3. Méndez F, Carrasquilla G, Muñoz A. Risk factors associated with malaria infection in an urban setting. Trans R Soc Trop Med Hyg 2000;94:367-71.

4. Méndez F. Epidemiología de la malaria en el Valle del Cauca 1987-1998. Cali: Boletín Epidemiológico Departamento del Valle del Cauca. Gobernación del Valle del Cauca; 1999;1:1-11.

5. Drummond MF, Sculpher MJ, Torrance GW, O'Brien BJ, Stoddart GL. Methods for the economic evaluation of health careprogrammes. Third Edition. Oxford: Oxford Medical Publications; 2005.

6. DANE. Resumen Nacional Censo 1993. Sistema de consulta del XVI Censo Nacional de Población y Vivienda. Santafé de Bogotá: Departamento Nacional de Estadística; 1996.
7. Pyndick R, Rubinfield D. Econometric models and economic forecasts. 4th edition: New York: Mc GrawHill; 1997.

8. Sheskin D. Handbook of parametric and nonparametric statistical procedures. Second edition: Boca Ratón, FL: CRC Press; 2000.

9. Rojas W, Botero S, García H. An integrated malaria control program with community participation on the Pacific Coast of Colombia. Cad Saúde Publica 2001;17:103-13.

10. Sharma SN, Saxena NB, Phukan PK, Anjan JK, Pandya AP, Lal S. Impact assessment of IEC campaign during anti-malaria month, June 1998 through KABP study. J Commun Dis 2000;32:49-53.

11. Kroeger A, Meyer R, Mancheno M, González M. Health education for community-based malaria control: an intervention study in Ecuador, Colombia and Nicaragua. Trop Med Int Health 1996;1:836-46.

12. Marsh VM, Mutemi W, Some ES, Haaland A, Snow RW. Evaluating the community education programme of an insecticide-treated bed net trial on the Kenyan coast. Health Policy Plan 1996;11:280-91.

13. Konradsen F, van der Hoek W, Amerasinghe PH, Amerasinghe FP. Measuring the economic cost of malaria to households in Sri Lanka. Am J Trop Med Hyg 1997;56:656-60.

14. Attanayake N, Fox-Rushby J, Mills A. Household costs of 'malaria' morbidity: a study in Matale district, Sri Lanka. Trop Med Int Health 2000;5:595-606.

15. Ettling MB, Shepard DS. Economic cost of malaria in Rwanda. Trop Med Parasitol 1991;42:214-8.

16. Sauerborn R, Shepard DS, Ettling MB, Brinkmann U, Nougtara A, Diesfeld HJ. Estimating the direct and indirect economic costs of malaria in a rural district of Burkina Faso. Trop Med Parasitol 1991;42:219-23.

17. Asenso-Okyere W, Dzator J. Household cost of seeking malaria care. A retrospective study of two districts in Ghana. Soc Sci Med 1997;45:659-67.

18. Ruíz W, Kroeger A. The socio-economic impact of malaria in Colombia and Ecuador. Health Policy Plan 1994;9:144-54. 